Volume 10, No.4, July - August 2021

International Journal of Advanced Trends in Computer Science and Engineering

Available Online at http://www.warse.org/IJATCSE/static/pdf/file/ijatcse081042021.pdf

https://doi.org/10.30534/ijatcse/2021/081042021

\title{
Density based Traffic Control with Alert System using Arduino and NodeMCU
}

\begin{abstract}
Sai Varshitha Rodda ${ }^{1}$, Sai Srinidhi Karanam ${ }^{2}$, KC Sriharipriya ${ }^{3}$
Undergraduate student, Vellore Institute of Technology, Vellore, India, saivarshitha.rodda2018@vitstudent.ac.in Undergraduate student, Vellore Institute of Technology, Vellore, India, karanamsai.srinidhi2018@vitstudent.ac.in School of Information Technology, Vellore Institute of Technology, Vellore, India, sriharipriya.kc@ vit.ac.in
\end{abstract}

\begin{abstract}
The aim of this paper is to design and implement a density-based traffic control system with automatic intruder identification using IoT. This system enables it to identify the density of the traffic at the dense traffic roads and accordingly adjust the timing of traffic signals in order to reduce the congestion at the road and also alerts when the vehicles cross the signal when 'red'. Hardware design is implemented by using Arduino and NodeMCU. It also uses IR sensors that indicate traffic congestion at intersections (crossroads). The programming is done using Arduino IDE. The data is monitored on device and the image is displayed on the app along with time and date of the violation incident. This approach to traffic congestion is expected to significantly improve traffic congestion in order to predict traffic congestion and find a solution. It can also provide information about traffic violations and thus, help monitor and control effectively.
\end{abstract}

Keywords - Arduino Uno, Internet of Things(IoT), ThingSpeak, IR sensor, Arduino.

\section{INTRODUCTION}

2.

One of the most common problems across the world is getting stuck in a traffic jam. As the population is increasing day by day, the number of vehicles are increasing which causes road traffic congestion. The daily commuting on the roads is becoming more exhausting every day, leading to the failure of the public transportation system. To keep in pace with the growing developing activities in major cities, there is a need to have a smart traffic control system that manages the traffic light timings and detects traffic light violators. High volume of the vehicles, the inadequate infrastructure and the irrational distribution of the traffic signalling system are main reasons for this chaotic congestion. Therefore, in order to get rid of these problems or at least reduce them to a significant level, newer schemes need to be implemented by bringing in sensor based automation techniques in this field of traffic signalling system.

\section{PROPOSED SYSTEM}

System Overview - The objective of the system is to adjust the traffic light timings as traffic density on a particular road. If a traffic violation is done at a red light, then the control unit will receive the message that "Signal is crossed "and at what time.

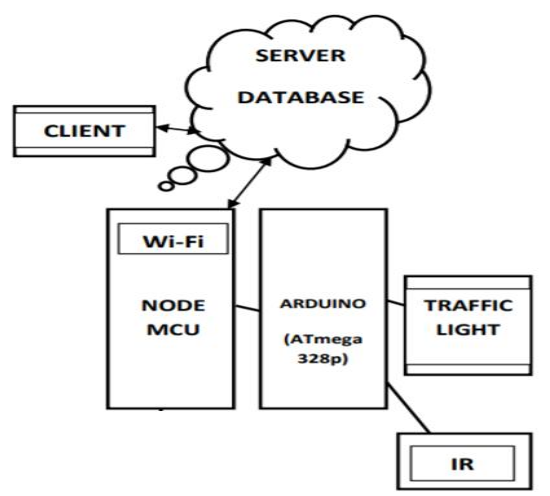

Figure 1: Block diagram of proposed system

\section{1 COMPONENTS USED}

Arduino - Arduino is an open-source project that created microcontroller-based kits for building digital devices and interactive objects that can sense and control physical devices.

ESP8266 NodeMCU - NodeMCU is an open-source firmware and development kit that helps to prototype and build IoT products. It includes firmware that runs on the ESP8266 Wi-Fi SoC from Espressif Systems, and hardware which is based on the ESP-12 module.

ThingSpeak - ThingSpeak is an IoT application platform providing various services exclusively targeted for building IoT applications. It offers capabilities like realtime data collection, visualizing the collected data in the form of charts and widgets, ability to create plugins and apps for collaborating with web services, social network and other APIs. 


\subsection{ALGORITHM}

The Arduino will receive information about the traffic density using IR sensors. Under Normal traffic conditions, the time duration of each traffic light (red, yellow, green) is two seconds.

Depending on the traffic movement received from the IR sensors, the data will be managed dynamically to avoid traffic congestion.

In case of traffic congestion, the IR sensors detect the presence of the vehicle and send the density information to Arduino UNO which alters the duration of green traffic light of the particular lane from two seconds to six seconds.

When there is no traffic violation, the density management is done as per the specified flow, i.e. two seconds each in case the traffic density decreases to normal and remains six seconds if heavy traffic still persists.

In case of traffic violation, i.e. if a vehicle jumps the red light,the data reaches the ThingSpeak Channel. Further, the control unit will receive the message that "Signal is crossed " and at what time.

The mobile app displays the date and time of violation along with the message. This can be accessed by the traffic administrator from any smart device such as laptop, desktop, smart phones, tablets, etc.

\subsection{FLOW CHART}

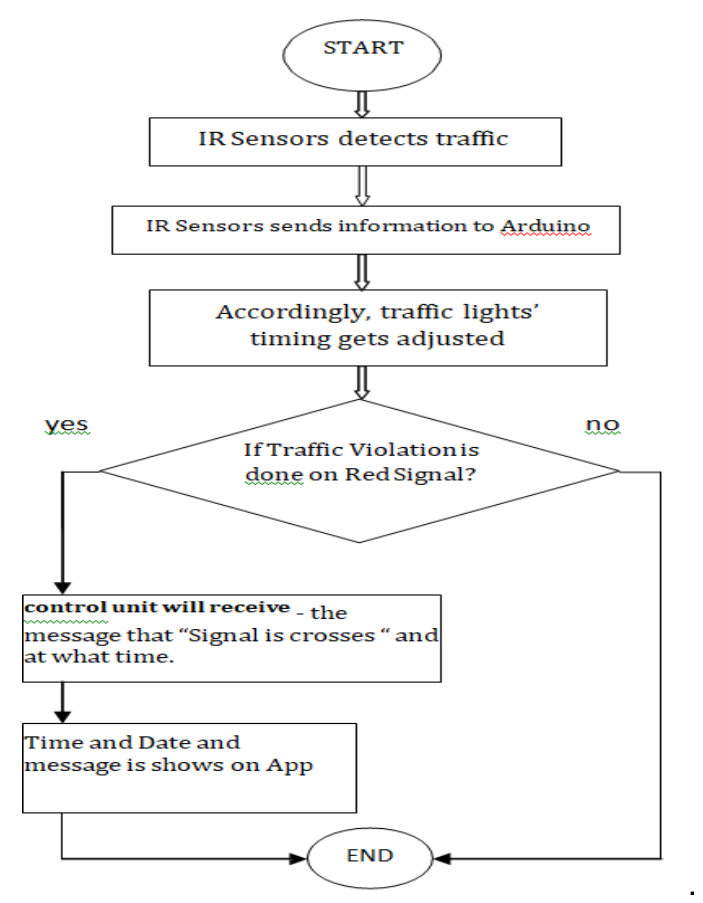

Figure 2: Flow chart

\section{RESULTS AND DISCUSSIONS}

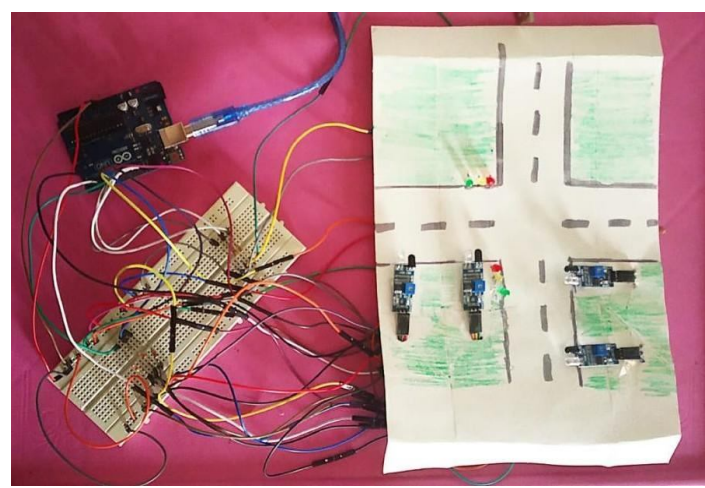

a) Hardware

Figure 3: Hardware setup

b) ThingSpeak channel

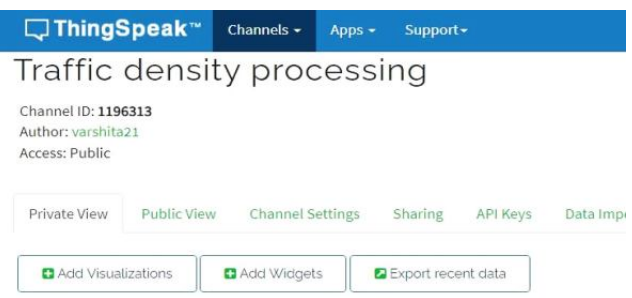

Channel Stats

Created: .7.days.ago Last entry: 8.minutes.ago
Entries: 11

\section{Field 1 chart}

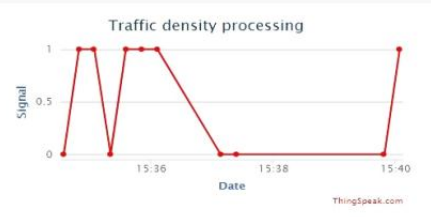

Figure 4: ThingSpeak channel

c) Smartphone notification

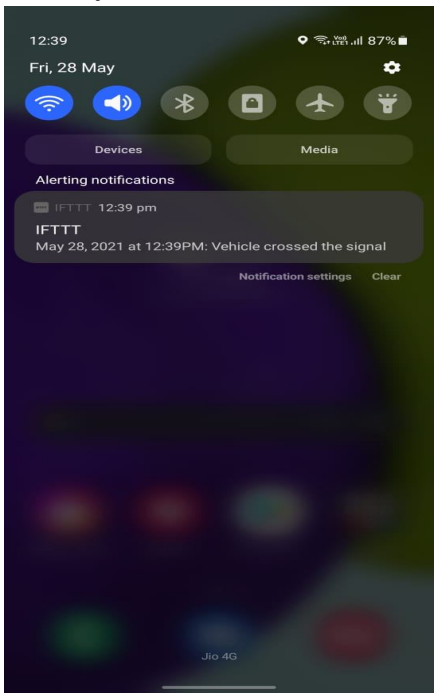

Figure 4: Smartphone alert 


\section{CHALLENGES AND FUTURE SCOPE OF ADVANCEMENTS}

Although the prototype model worked very efficiently with remarkable outputs, the real-time situation is going to be way more demanding and challenging. Few of the challenges that should be taken into consideration are listed as follows

- Low range IR sensors may not be suitable for long range traffic signalling systems. We may convert to ultrasound or radar techniques for big scale set-ups.

- Periodic checking of the precision and accuracy is a must for efficient operation of this model prototype.

- Next is the influence of drift signals that may differ the reading of sensory receptors and lead to conveying any false information to the microcontroller.

Safety first: It has to be absolutely made sure that no compromise is being made on the safety issues, i.e. a secondary stand-by set-up that can switch from automated to the manual mode, should be accommodated in the case of circuit or sensor malfunctions so that vehicular crowd do not go beyond control. As part of future work and advancements, the traffic check post can be connected by wireless transmitters by which the crossings ahead may be predicted of the traffic that is approaching. This may be achieved by connecting the sensor network with GPS connectivity and short-wave radio transmission signals. This will act as a feed forward system making the signalling system even more smooth and congestion free. We will also update this system with modern technologies so that when a vehicle tries to move even during a red signal it will turn on an alarm in the form of an alert to warn the driver of the vehicle.

\section{CONCLUSION}

Arduino UNO and NodeMCU (ESP826612E) were used to develop a density based traffic controller along with an integrated camera module for default identification. Upon detecting heavy congestion, the IR sensors send the related information to Arduino which manipulates the 'Green' time of traffic lights to allow the passage of the traffic. The defaulter, who skips the red light is also captured using a NodeMCU and sending a message. We designate ESP826612E as an access point and use it to make a web server which displays messages captured during violation time and date on the app accessed by the user. Thus, from above results or outputs, we can conclude that using the method of density-based control of traffic lights we can save a considerable amount of time and also we can prevent excessive traffic jams thus leading to smooth traffic flow. In practice presently in India we are following time-based traffic signal control and we are experiencing heavy traffic jams all over which in turn consumes a lot of time and fuel due to unnecessary waiting time. We hope this method will be adopted as soon as possible so that the limitations we are experiencing with present methods can be overcome.

\section{REFERENCES}

[1] Aaron Don M. Africa, Francis Xavier Asuncion, Janos Lance Tiberio, Raymund Miguel Francisco A. Munchua "Sensor-based Traffic Control Network with Neural Network Based Control System"IJATCSE Vol 8 (August 2019).

[2] Mr. Bilal Ghazal, Mr. Khaled ElKhatib"Smart Traffic Light Control System"- IJERT (April 2016) [3] Mr. Shubham sahu, Mr. Depanjampaul, Mr. Senthilmurgan"Density based traffic control using IR sensors"- IRJET (June 2019).

[4] Mr. Piyush mahajan, Ms. punamkumari, Mr. HPS kang"Design and implementation of portable traffic system"- IJRTER (2017).

[5] Mr. Tenepallikalyan sai manikantachari, Mr. Sai tejatutika"Density based traffic control signaling using ir sensor"-- Philpapers (2019). 